Special issue of the 2nd International Conference on Computational and Experimental Science and Engineering (ICCESEN 2015)

\title{
Sharp-Rejection, Wide and Deep Stopband Low Pass Filter Design Using Open Stubs and DGS Patterns
}

\author{
M. Challal ${ }^{a, *}$ AND M. BoulakrounE ${ }^{b}$ \\ ${ }^{a}$ University M'Hamed Bougara of Boumerdes, Institute of Electrical and Electronic Engineering, Signals and \\ Systems Laboratory, Department of Electronics, Boumerdes, Algeria \\ ${ }^{b}$ University Kasdi Merbah of Ouargla, Faculty of New Technologies of Information and Communication, Electronics \\ and Communication Department, Ouargla, Algeria
}

\begin{abstract}
A sharp rejection, wide and deep stopband microstrip low pass filter is investigated in this paper. The proposed low pass filter is composed of three defected ground structure patterns and four open stubs. Simulated results verify that the filter has a sharpness factor of 0.9 , a low insertion loss of 0.65 and a return loss less than $15 \mathrm{~dB}$. Moreover, a wide stopband at $20 \mathrm{~dB}$ rejection ranging from $4.55 \mathrm{GHz}$ to $11.62 \mathrm{GHz}$ is accomplished. The filter presented in this work was compared with the previous works completed on low pass filter with sharp rejection. It has shown that the filter provides good performances in terms of sharp rejection, wide and deep rejection band and small passband insertion loss compared to those reported in the literatures.
\end{abstract}

DOI: 10.12693/APhysPolA.130.9

PACS/topics: 41.20.-q, 41.20.Jb, 11.10.-Z

\section{Introduction}

Filters of high quality, portability and flexible reconfiguration are always desirable in modern and wireless communication systems such as radar, satellite and mobile communications to remove undesired harmonics or spurious mixing products. Low-pass filters (LPFs) based on defected ground structure (DGS) patterns have been proposed for a particular requirement and application [1,2].

In this paper, a modified LPF with sharp transition band, wide and deep stop-band is investigated based on DGS patterns, proposed by Wang [3], and open stubs. A parametric study is conducted to show the frequency characteristics of the DGS and then, a LPF with sharp rejection, wide and deep stopband is designed. All structures are designed and analyzed using an electromagnetic simulator based on the method of moment. The performances of the proposed LPF are compared with those reported in literature.

\section{Design concept}

Figure 1 shows the geometry of the DGS cell proposed in [3]. In this study, all structures are printed on a dielectric substrate with a relative permittivity of $\varepsilon_{\mathrm{r}}=3.38$ and a thickness of $h=0.813 \mathrm{~mm}$. Dielectric and metallic losses, as well as the finite thickness of the metallic layers were taken into account during the design. The width of the $50 \Omega$ transmission line is $1.87 \mathrm{~mm}$ whereas the DGS cell dimensions $l_{1}, d, L$, and $w$, are respectively $2,3,6.9$, and $1 \mathrm{~mm}$.

\footnotetext{
* corresponding author; e-mail: mchallal@umbbdz
}

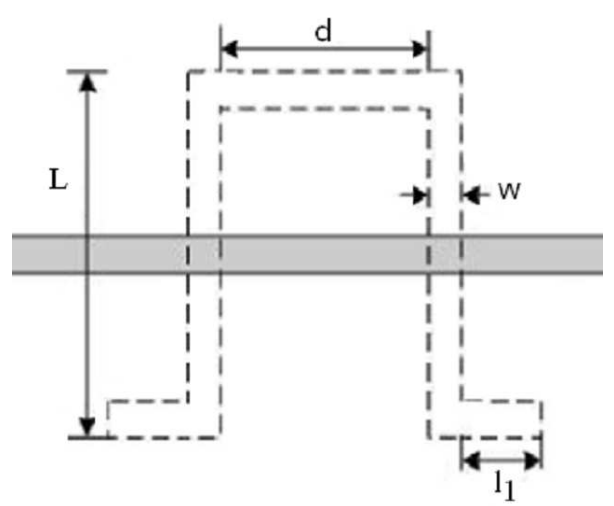

Fig. 1. Geometry of the DGS cell [3].

The sharpness factor (SF) is a reference to check whether the filter is considered sharp or not and it is expressed as follows:

$$
\mathrm{SF}=f_{\mathrm{c}} / f_{0},
$$

where $f_{\mathrm{c}}$ and $f_{0}$ are respectively the cut-off and the attenuation pole frequencies.

The effect of the dimensions $w, L, l_{1}$, and $d$ on the DGS cell performance, shown in Fig. 1, is investigated. Figure 2 shows the $S_{21}$-parameter for different values of $L$ when the other parameters are kept constant.

Referring to Fig. 2 , as $L$ increases, $f_{\mathrm{c}}$ and $f_{0}$ decrease while $S_{21 \text { max }}$ increases, but $S_{21 \text { max }}$ starts to decrease for the last two values $(6.9 \mathrm{~mm}$ and $7.9 \mathrm{~mm})$. The length $L$ is chosen to be $6.9 \mathrm{~mm}$ (because of $\mathrm{SF}=0.92$ ) and, $S_{21 \max }$ at $L=6.9 \mathrm{~mm}$ is greater than that at $L=7.9 \mathrm{~mm}$. Similarly, an increase in $d$, from $1 \mathrm{~mm}$ to $4 \mathrm{~mm}$ with step of $1 \mathrm{~mm}$, results in a decrease in $f_{\mathrm{c}}, f_{0}$ and SF whereas $S_{21 \max }$ increases. The chosen dimension of $d$ is $3 \mathrm{~mm}$ (because of its compact size) and $\mathrm{SF}=0.92$ while that at $d=4 \mathrm{~mm}$ is 0.91 and they have almost the same $S_{21 \max }$. 


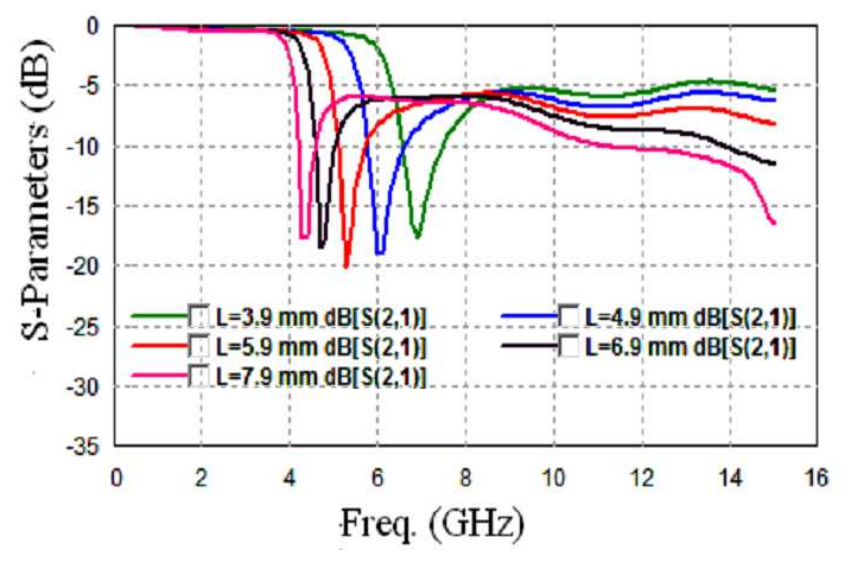

Fig. 2. $S_{21}$-parameter for different values of $L$.

In the same way, an increase in the length of $l_{1}$ causes a decrease in $f_{\mathrm{c}}, f_{0}$ and $S_{21 \max }$ while the SF increases very slowly so $l_{1}$ is taken to be $2 \mathrm{~mm}$. Also, an increase of $w$ has almost no effect on $f_{\mathrm{c}}$ and $f_{0}$ while $S_{21 \text { max }}$ increases significantly. The suitable value of $w$ that can be chosen for this DGS cell is $1 \mathrm{~mm}$. The main objective is to design an LPF with sharp rejection, wide and deep stopband. By comparing the obtained results, it can be concluded that the best dimensions of the DGS cell are: $w=1 \mathrm{~mm}$, $d=3 \mathrm{~mm}, l_{1}=2 \mathrm{~mm}$ and $L=6.9 \mathrm{~mm}$. The choice of these dimensions is based on small $f_{\mathrm{c}}$, high $\mathrm{SF}$ and passband return loss below $-13 \mathrm{~dB}$.

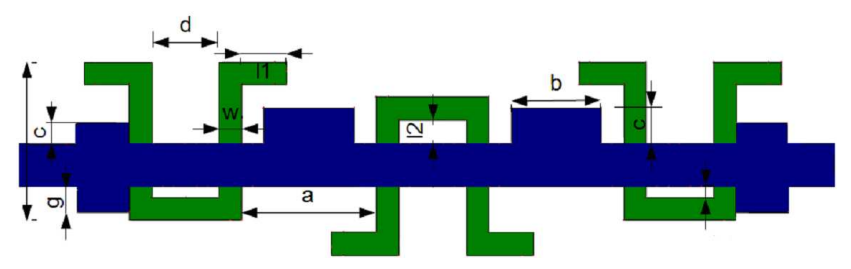

Fig. 3. The proposed LPF based on DGS units and open stubs.

To improve the characteristics of the LPF, more DGS units were added to form what is called periodic DGS to achieve best results for sharp rejection and wide stopband. The design is conducted starting from two DGS units, three DGS units and then three DGS with open stubs. After an optimally design process for the quest of a sharp rejection and a wide stopband microstrip LPF, two open stubs are introduced between the three DGS units and two other open stubs are added near the end of each DGS units (right and left ones only), as shown in Fig. 3. The optimal dimensions $a, b, c, g, L, l_{1}, l_{2}, l_{3}$, $d$, and $w$, are respectively $5,4,0.5,1,6.9,2,1,0.5,3$, and $1 \mathrm{~mm}$. The simulation result of the proposed LPF structure is shown in Fig. 4.

It is clearly that the proposed LPF is sharp and has a wide stopband at $20 \mathrm{~dB}$ rejection ranging from $4.55 \mathrm{GHz}$ to $11.62 \mathrm{GHz}$. The filter presented in this work has been compared with the previous works done on LPF with

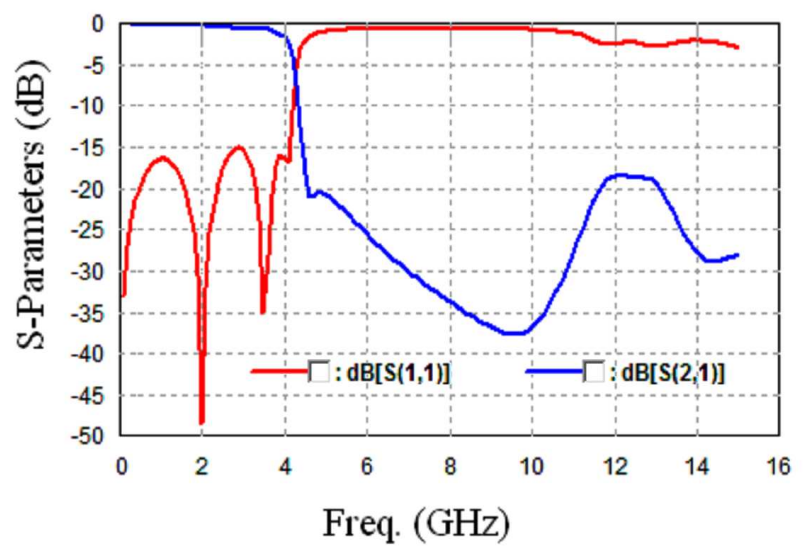

Fig. 4. $S$-parameters of the proposed LPF.

sharp rejection. The performance of the proposed LPF is summarized in Table I with other reported LPFs for comparison.

TABLE I

Comparison of the proposed DGS-LPF with other related LPF.

\begin{tabular}{c|c|c|c}
\hline \hline Characteristics & {$[4]$} & {$[5]$} & This work \\
& 2007 & 2008 & \\
\hline substrate material $\left(\varepsilon_{\mathrm{r}} / h\right)$ & $2.65 / 0.254$ & $2.2 / 0.787$ & $3.38 / 0.813$ \\
cutoff frequency $f_{\mathrm{c}}[\mathrm{GHz}]$ & 3.8 & 2.00 & 4.14 \\
stopband [dB] & $4.3-6.5$ & $3.1-7.9$ & $4.55-11.62$ \\
at 20 dB rejection & & 0.20 & $<, 0.65$ \\
passband insertion loss [dB] & $<0.9$ & 0.55 & 0.9 \\
sharpness factor [SF] & 0.86 & 0.5
\end{tabular}

From Table I, it can be seen that the proposed microstrip LPF provides good performances in terms of sharp rejection, wide and deep rejection band and, small passband insertion loss than those reported in literature $[4,5]$.

\section{Conclusion}

In this paper, an LPF with sharp transition band, wide and deep stopband has been introduced. The proposed microstrip LPF has a sharpness factor of 0.9 , an insertion loss and a return loss less than 0.65 and $15 \mathrm{~dB}$, respectively. In addition, a wide stopband at $20 \mathrm{~dB}$ rejection ranging from $4.55 \mathrm{GHz}$ to $11.62 \mathrm{GHz}$ is achieved.

\section{References}

[1] M. Challal, A. Boutejdar, Microwave Opt. Technol. Lett. 55, 122 (2013).

[2] M. Challal, A. Boutejdar, ACES J. 27, 808 (2012).

[3] Wang Huina, Li Guohui, Yan Jun, in: Proc. ChinaJapan Joint Microwave Conference (CJMW 2008), IEEE, Shanghai (China) 2008, p. 388.

[4] J. Zhang, B. Cui, S. Lin, X.-W. Sun, Prog. Electromagn. Res. 69, 219 (2007).

[5] C.Y. Hung, M.H. Weng, R.Y. Yang, Microwave Opt. Technol. Lett. 50, 894 (2008). 